Epidemiology and Infection

cambridge.org/hyg

\section{Letter to the Editor}

Cite this article: Longo D, Narese D, Fariello $G$ (2018). Diagnosis of brain abscess: a challenge that Magnetic Resonance can help us win!. Epidemiology and Infection 146, 1608-1610. https://doi.org/10.1017/S0950268818001139

Author for correspondence:

Donatella Narese, E-mail: donatella.narese@ virgilio.it

\title{
Diagnosis of brain abscess: a challenge that Magnetic Resonance can help us win!
}

Daniela Longo ${ }^{1}$, Donatella Narese $\mathrm{D}^{1,2}$ and Giuseppe Fariello ${ }^{3}$

${ }^{1}$ Department of Imaging, Bambino Gesù Children's Hospital, IRCCS, Rome, Italy; ${ }^{2}$ Department of Radiology, DIBIMED, University of Palermo, Palermo, Italy and ${ }^{3}$ Fatebenefratelli S. Pietro Hospital, Rome, Italy

To the Editor: We read with enormous interest the Italian multicentre study by Raffaldi $e t$ al. [1], promoted by the Italian Society for Pediatric Infectious Diseases, describing retrospectively collected patients aged 0-18 years, with a diagnosis of 'brain abscess' (BA).

As the multicentre study has well highlighted, brain infections in children can cause irreversible neurological damage and long-term neurodevelopmental impairment. Clinical signs are usually non-specific, and this is especially true for newborns, signalling towards a rapidly deteriorating infant; only rarely neonates present focal neurological signs. The authors conclude that the presentation with non-specific signs and symptoms could explain the delay in the diagnosis (about 7 days). Moreover, CSF (cerebrospinal fluid) analysis is frequently normal or non-contributory and, as the authors have well emphasised, lumbar puncture is not recommended because it can result in transforaminal or transtentorial herniation and consequent death.

Finally, focusing on neuroimaging techniques, the authors conclude that computed tomography (CT) or magnetic resonance imaging (MRI), allows one to confirm BA diagnosis and determine the location and the number of lesions. We are in complete agreement with their conclusions regarding the difficulty in diagnosis of this infrequent condition leading to the use of aggressive diagnostic methods.

Unfortunately, we must say that in the neonates, bedside transfontanellar ultrasound (US), in many hospital centres, remain the first-line modality of imaging because of their safety, low cost and especially because of their wide availability. This behaviour is an aggravating factor because screening with US rarely yields results that strongly suggest a cerebral infection [2-4]. On this aspect, we would like to share our experience with three similar cases of children whom, during their stay in the neonatal intensive care units (NICU), presented worsened general conditions:

Case 1. 37-week newborn. Persistent and severe hypoglycaemia, vomiting, diarrhoea with malodorous stools, acidosis and fever. High values of $\mathrm{C}$ reactive protein (CRP), procalcitonin (PCT) and white blood cells (WBC) count.

Case 2. 26-week newborn. On 18th-day general conditions worsened. High values of CRP and PCT.

Case 3. 37-week newborn. Prenatal diagnosis of multiple intestinal atresia; post-natal hypoxic-ischaemic encephalopathy (HIE); more than one surgery to correct bowel atresia during the first week of life. During his stay in the NICU seizures and lethargy. High values of CRP, PCT and WBC count.

All patients underwent a transfontanellar US scan that showed, increased echogenicity of the subcortical white matter and, in Case 3, extensive cystic periventricular leukomalacia, recognised as HIE sequelae. Only the further clinical deterioration induced neonatologists to require the confirmation of brain US findings by MRI evaluation. MRI features are consistent with cerebral abscesses in all cases (Figs 1-3). In our experience, the confirmation of brain US findings by MRI evaluation is very helpful, especially when US usually does not allow a perfect distinction between the site of a previous haemorrhage and an abscess [5].

Modern-day imaging techniques such as CT and MRI allow the prompt confirmation of the clinical diagnosis [6]. CT is commonly used for diagnosing abscesses in adults, because of its general availability and high specificity after contrast enhancement; it is more useful in older children than in neonates, in whom high water content of the brain reduces the contrast between normal and injured tissue. Diffusion-weighted MRI and post-contrast MRI have been gaining increasing importance in the evaluation of brain infections and abscesses because of its high sensitivity and specificity $[7,8]$.

In conclusion, considering the difficulty in diagnosis of this infrequent condition, we emphasise the need for standardised national guidelines or adequate recommendations not only on antibiotic treatment but also on diagnostic methods. 


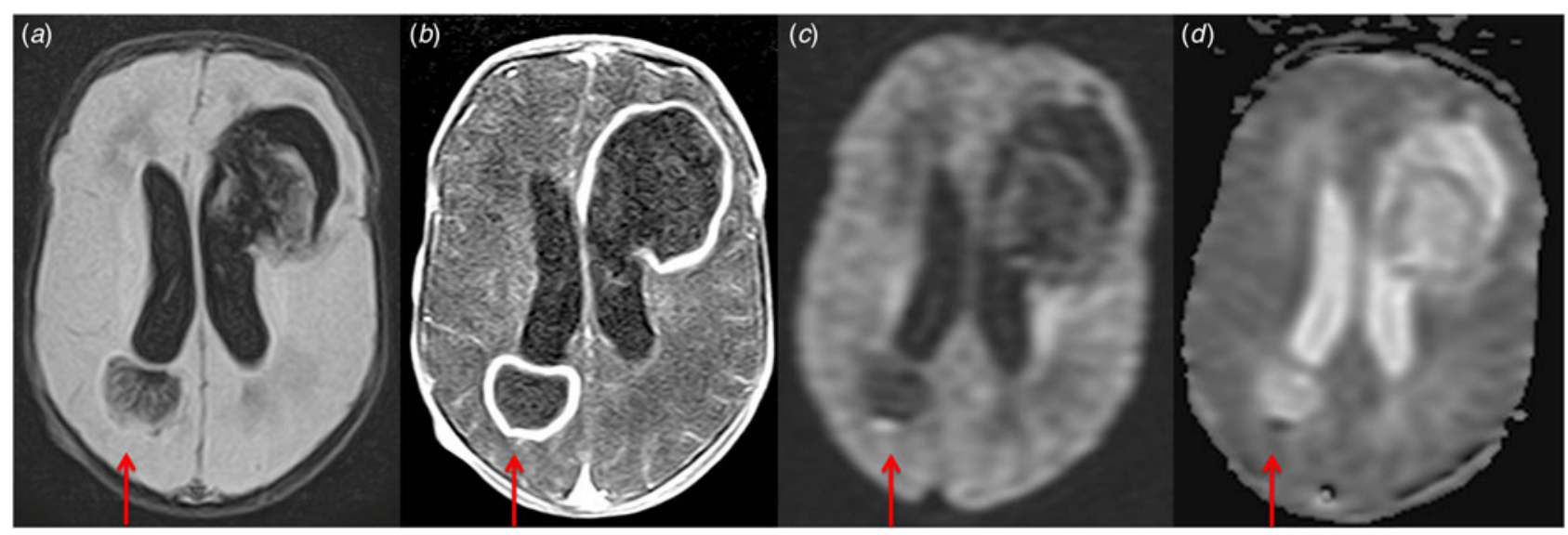

Fig. 1. Presence of two focal lesions in frontal and temporoparietal lobes with a hyperintense on fluid attenuation inversion recovery (FLAIR) images (a) with a hypointense rim with ring enhancement (b). The central core of the masses restricts strongly on the diffusion-weighted imaging (DWI) (c) and apparent diffusion coefficient map (ADC) (d). MRI features are consistent with cerebral abscesses.

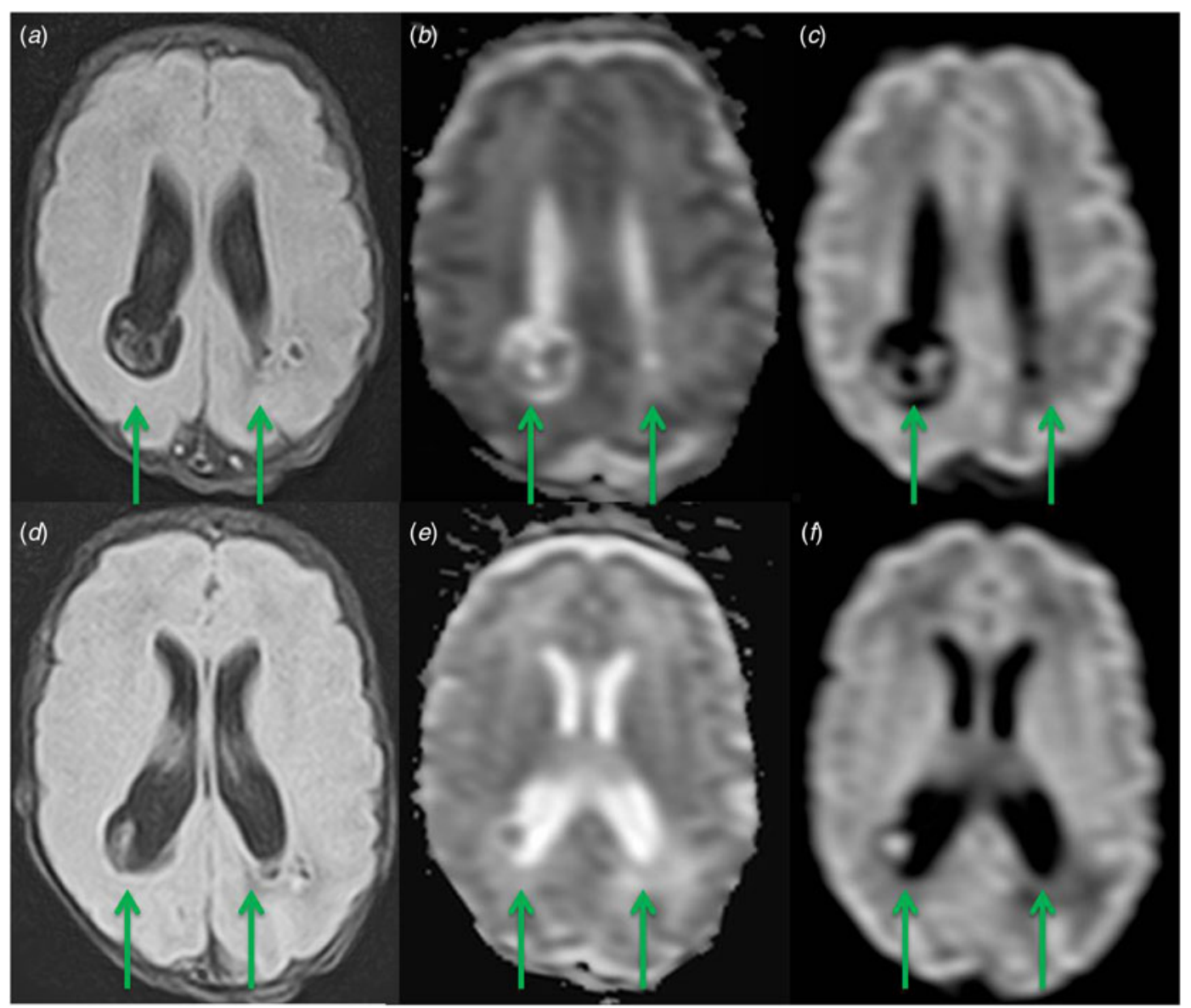

Fig. 2. Presence of multiple focal lesions in the parietal lobes, with a hyperintense centre and a mildly hypointense rim on FLAIR images (a, d). The central core of the masses restricts strongly on DWI (b, e) and ADC (c, f). MRI features are consistent with cerebral abscesses. 

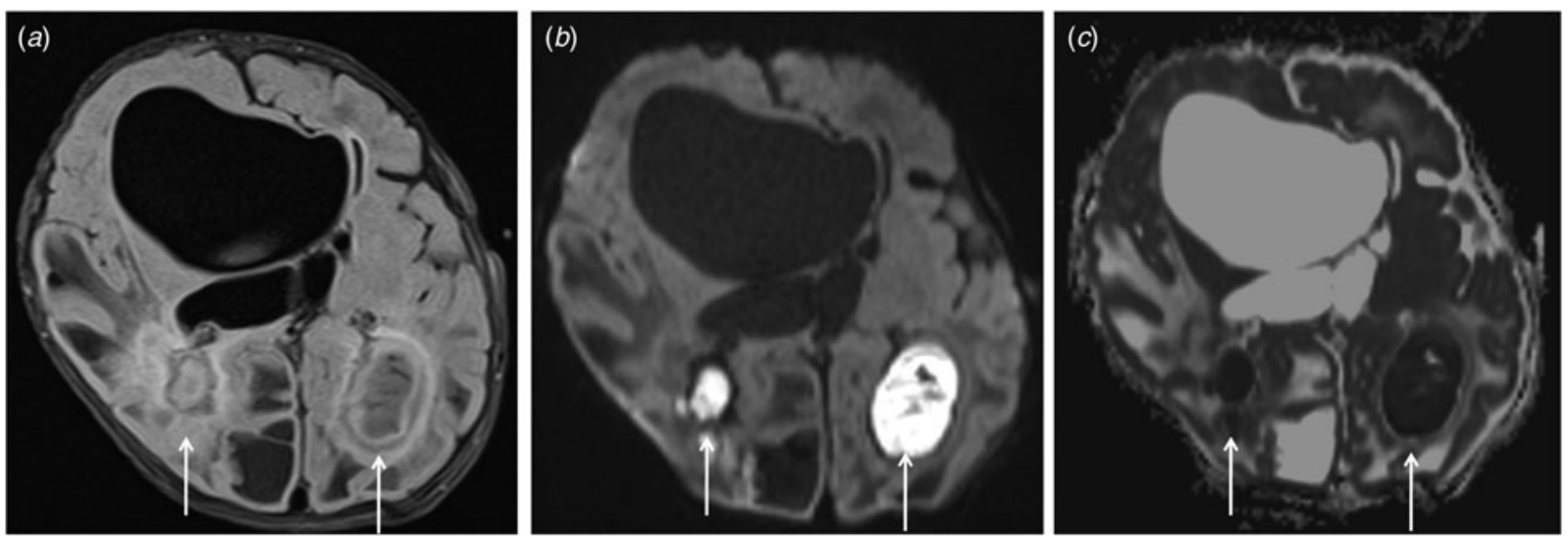

Fig. 3. Severe periventricular cystic changes around the bodies of the lateral ventricles. Presence of two focal lesions in the occipital lobes, with a hyperintense centre and a mildly hypointense rim on FLAIR images (a). The central core of the masses restricts strongly on DWI (b) and ADC (c). MRI features are consistent with cerebral abscesses.

\section{References}

1. Raffaldi I et al. (2017) Brain abscesses in children: an Italian multicentre study. Epidemiology and Infection 145, 2848-2855.

2. Ries M et al. (1993) Brain abscesses in neonates: report of three cases. European Journal of Pediatrics 152, 745-746.

3. Nagle RC et al. (1986) Brain abscess aspiration in nursery with ultrasound guidance: case report. Journal of Neurosurgery 65, 557-559.

4. Lam AH et al. (1984) Intracranial Serratia infection in preterm newborn infants. American Journal of Neuroradiology 5, 447-451.
5. Hirooka T et al. (2007) Cerebral abscess caused by Serratia marcescens in a premature neonate. Arquivos de Neuro-Psiquiatria 65, 1018-1121.

6. Sheehan JP et al. (2008) Brain abscess in children. Neurosurgical Focus 24, E6.

7. Castillo M (1999) Imaging brain abscesses with diffusion-weighted and other sequences. American Journal of Neuroradiology 20, 11931194.

8. Ciurea AV et al. (1999) Neurosurgical management of brain abscesses in children. Child's Nervous System 15, 309-331. 